\title{
Handwritten Arabic Numeral Character Recognition Using Multi Kernel Learning Support Vector Machine
}

\author{
Muhammad Athoillah ${ }^{* 1}$, Rani Kurnia Putri ${ }^{2}$ \\ 1,2Universitas PGRI Adi Buana Surabaya/Department of Mathematics Education \\ athoillah.muhammad@gmail.com ${ }^{* 1}$, rani@unipasby.ac.id ${ }^{2}$
}

\begin{abstract}
Handwritten recognition is how computer can identify a handwritten character or letter from document, image or other sources. Recently, many devices provide a feature using handwritten as an input such as laptops, smartphones, and others, affecting handwritten recognition abilities become important thing. Recognizing Arabic character is a challenging task. As the prime language of Muslim people, the outcome of that recognizing system has to be quite accurate because the result of the process will impact on the entire process of understanding the Qur'an lesson. Basically, handwritten recognition problem is part of classification problem and one of the best algorithm to solve that problem is Support Vector Machine (SVM). By finding a best separate line and two other support lines between input space data in process of training, SVM can provide the better result than other classify algorithm. Although SVM can solve the classify problem well, SVM must be modified with kernel learning method to be able to classify nonlinear data. However, determining the best kernel for every classification problem is demanding. Therefore, some techniques have been developed, one of them is Multi Kernel Learning (MKL) which is processed by combining some kernel function to be one kernel with an equation. This framework built an application to recognize handwritten Arabic numeral character using SVM algorithm that modified with Kernel Learning Method. The result shows that the application can recognize data well with average value of Accuracy is $86.63 \%$.
\end{abstract}

Keywords: Arabic, Classification, Handwritten, Machine Learning, Pattern Recognition

\section{Introduction}

Study of text recognition have increased in recent years, due to increased number of its applications in image retrieval, scene understanding and also the growing number of many devices that can receive handwritten as an input such as smartphones, Televisions, Laptops etc. [1][2]. The number of that smart devices is believed to be much increased in future days which affects text recognition precisely handwritten text recognition abilities becomes important thing. The handwritten recognition is an ability of system to recognize and convert human writing into digital text. There are two kind of handwritten recognition that are on-line recognition and off-line recognition. The on-line handwritten recognition is a system that can recognize human handwriting directly from input device such as smartphones or personal digital assistant (PDA). Meanwhile the off-line handwritten recognition is a system that can recognize human written by scanning any images [3].

Recognizing Arabic character is a challenging task. Since it is the mother tongue of the Muslims and the only language used in their holy book AI Qur'an, the outcome of that recognizing system has to be quite accurate, the results of the process will impact on the entire process of understanding the Qur'an lesson. Aside from that important reason, the automatic recognition of Arabic handwritten characters is required due to some factors. The first factor is the lack of number Arabic programming tools and digital dictionaries, and also the absence of big public databases of Arabic handwritten characters and words if compared with English that having large database center such as CEDAR (Center of Excellence for Document Analysis and Recognition). Secondly the start of Arabic handwritten characters and words recognition is very late if compared to other languages such as Chinese and Latin [3].

Handwritten recognition had studied for the first time by T. L. Dimond by developed a device for reading handwritten characters in 1958 [4]. While, the first publication of Arabic text recognition had done by A. Nazif in 1975 [3]. Recently, due to the vast development of science, although not as fast as the growing number of Chinese and Latin character recognition study, the amount of

Athoillah, M., \& Putri, R. (2019). Handwritten Arabic Numeral Character Recognition Using Multi Kernel Support Vector Machine. Kinetik: Game Technology, Information System, Computer Network, Computing, Electronics, and Control, 4(2). doi:http://dx.doi.org/10.22219/kinetik.v4i2.724 
study of Arabic text recognition has also growing quite rapidly. For instance, Mohammed Ali et al presented Arabic Handwritten Recognition Using Very Deep Neural Network in 2017 [5], Jayech Khaoula et al in 2016 researched offline Arabic Handwritten Recognition using Multi-Steam Hidden Markov Model [6], Shatnawi M and Abdallah S who had improved Handwritten Arabic Character Recognition by Modeling Human Handwriting Distortions in 2016 [7], Rabi M et al presented Recognition of cursive Arabic handwritten text using embedded training based on HMMs in 2018 [8], and some other researchers. Most of that researches was studied about Arabic character recognition. So, almost no studies were found that talked about Arabic number recognition while the fact is both Arabic letters and numbers have striking differences.

Basically, the handwritten recognition problem is part of classification problem. In recent years, many classified algorithm has been proven helping people's works, such as image classification, medical biology, traffic light, and text classification [9]. One of that algorithm is Support Vector Machine (SVM). SVM is supervised algorithm that separating class of data by using three separating line, that is one main separating line and two other lines are support line, this makes SVM provide better result than other algorithms [9]. Although SVM can solve the classify problem well, SVM can only be used for linear separable data, to be able to classify nonlinear data, SVM must be modified with kernel learning method. The kernel method makes SVM can map data into a higher dimensional vector space, thus in the new vector space, the separating line can be constructed [10]. Nevertheless, selecting the best kernel to solve the classification problem well is quite difficult since every kernel does not provide best result for every classification problem. This is because every data that use for each problem has its own characteristic. Consequently, some techniques have been developed by researcher to solve the difficulty for instance making more flexible kernel function. One of those method is Multi Kernel Learning (MKL). This technique works by combining some kernel function to be one kernel's equation. Lots of recent research have shown that the application of multi kernel in SVM algorithm has better performance than the use of single one [11] [12].

Based on that's background, this framework built an application which is able to recognize handwritten Arabic numeral character from an image using Support Vector Machine algorithm which is modified with Kernel Learning Method. This framework used image dataset from MADBase dataset from The American University in Cairo, this database is contain handwritten numerical zero to nine in Arabic character. This, frameworks also provide the comparison result between our proposed method with single kernel method such as RBF and Polynomial kernel.

\section{Research Method}

\subsection{Support Vector Machine for Classification}

Support Vector Machine (SVM) is one the best algorithm to solve classification problem. Different from other algorithm that usually find the best single line between classes to classify data. SVM is supervised algorithm separating class of data by using three separating lines, one for main separating line and two other lines are support line (Figure 1), this is the reason SVM provides better result than other algorithms [9]. To find the best separating line in SVM, firstly find the two-supporting line between two classes following Equation 1 and 2.

$$
\begin{aligned}
& x_{i} \cdot w+b \geq+1 \text { for } y_{i}=+1 \\
& x_{i} \cdot w+b \geq-1 \text { for } y_{i}=-1
\end{aligned}
$$

Which $b$ is the relative position to the center coordinates and $w$ is the normal space, while data on the two-support line is called support vector.

The best main separating line is obtained by maximizing the margin between that twosupport line, it can be found by solving the following optimization problem (Equation 3 and 4) [13].

$$
\min \frac{1}{2}\|w\|^{2}
$$

Subject to

$$
y_{i}\left(x_{i} \cdot w+b\right)-1 \geq 0
$$

KINETIK Vol. 4, No. 2, May 2019: 99-106 
Lagrange multipliers and Karush-Kuhn-Tucker (KKT) is used to solve that optimization problem [13]. When the optimization problem is solved, then the class of data $x$ can be determined based on this Equation 5.

$$
f\left(x_{d}\right)=\sum_{i=1}^{m s} \alpha_{i} y_{i} x_{i} x_{d}+b,
$$

Where $x_{i}$ is support vector, $\mathrm{ms}=$ support vector number and $x_{d}$ is data that will be classified.

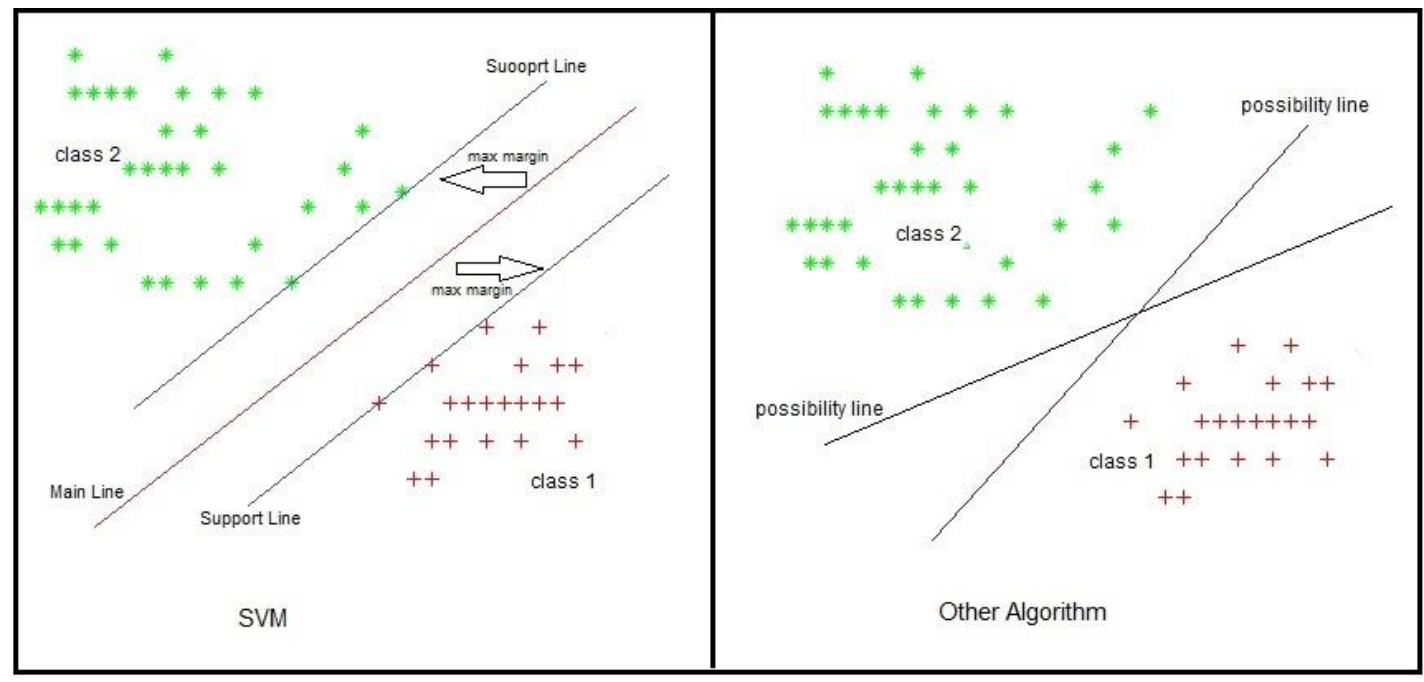

Figure 1. Different SVM with Another Algorithm

\subsection{Multi Kernel Support Vector Machine}

Although the Support Vector Machine can solve the classification problem well, SVM can only be used for linear separable data. In order to classify nonlinear data, SVM must be modified with kernel learning method. Furthermore, to classify nonlinear data, first, data have to be map into a higher dimensional vector space with function $\Phi$ so $\Phi: x \rightarrow \Phi(x)$ [10]. Thus, the separating line can be constructed in the new vector space (Figure 2).

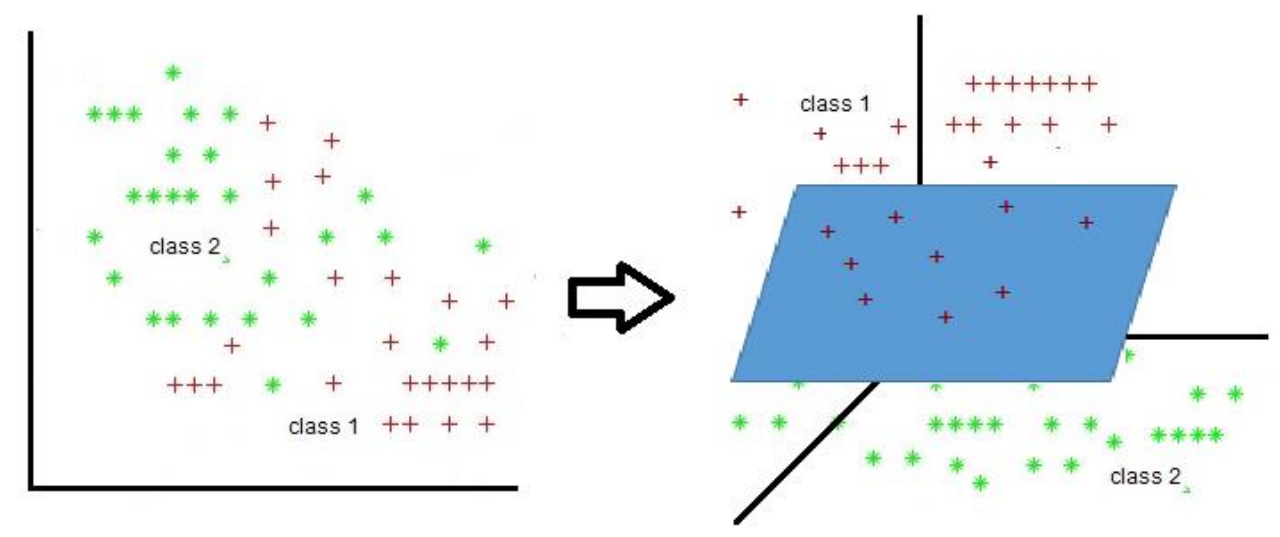

Figure 2. Mapping Data to Higher Dimensional

However, finding the transformation function $\Phi$ is a difficult work. Then to simplify it, the dot calculation can be replaced with a kernel function $K\left(x_{i}, x_{j}\right)$ [10]. Using the given formulation below (Equation 6).

$$
K\left(x_{i}, x_{j}\right)=\left\langle\Phi\left(x_{i}\right) . \Phi\left(x_{j}\right)\right\rangle
$$


This trick determines the support vector during the process of training SVM become much easier. After modified with kernel function, the equation to determine class data $x$ (decision function equation) can be written by the following Equation 7 .

$$
\begin{aligned}
& f\left(x_{d}\right)=\sum_{i=1}^{m s} \alpha_{i} y_{i} x_{i} x_{d}+b \\
= & \sum_{i=1, x_{i} \in S V}^{m s} \alpha_{i} y_{i}\left\langle\Phi\left(x_{i}\right) . \Phi\left(x_{d}\right)\right\rangle+b \\
= & \sum_{i=1, x_{i} \in S V}^{m s} \alpha_{i} y_{i} K\left(x_{i}, x_{d}\right)+b
\end{aligned}
$$

Determining which kernel is the best one for every classification problem is challenging. Because every classification data that use for each problem has its own characteristic. For that reason, there are some techniques that have been developed by researchers to solve that difficulties, one of them is Multi Kernel Learning (MKL). Many recent researches have shown that the use of multi kernel in SVM algorithm has better performance than the use of single one [11] [12]. Multi Kernel Learning technique works by combining some kernel functions to be one kernel's equation. Suppose it is given some single kernel function $K_{1}\left(x, x^{\prime}\right), K_{2}\left(x, x^{\prime}\right), \ldots, K_{N}\left(x, x^{\prime}\right)$ then the combination of all the kernels can be written as Equation 8 and 9.

$$
K\left(x, x^{\prime}\right)=\sum_{n=1}^{N} d_{n} K_{n}\left(x, x^{\prime}\right)
$$

Subject to

$$
d_{n} \geq 0, \sum_{n=1}^{N} d_{n}=1
$$

Which $N$ is number of kernel function [11]. Using this multi kernel equation, the decision function Equation 10.

$$
f\left(x_{d}\right)=\sum_{i=1, x_{i} \in S V}^{m} \alpha_{i} y_{i} \sum_{n=1}^{N} d_{n} K\left(x_{i}, x_{d}\right)+b
$$

\section{Results and Discussion}

\subsection{Implementation}

The dataset that is used in this framework is taken from MADBase dataset from The American University in Cairo [14]. This framework (Figure 3) used an image dataset containing handwritten numerical zero to nine in Arabic character with 500 number of each image in total. It means that 5000 images for all number which is 90 percent for data training and 10 percent for testing.

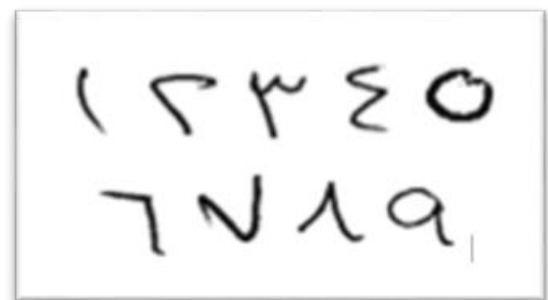

Figure 3. Sample of Dataset Images

KINETIK Vol. 4, No. 2, May 2019: 99-106 
In Figure $4 \mathrm{~K}$-fold cross validation method is used to validate the application that has been built in this framework. The image dataset is divided into ten groups $(k=10)$, that is nine of they are used for training process and any other is used for testing process, then the process is repeated $k$ times which is training and testing data are always different during each process.

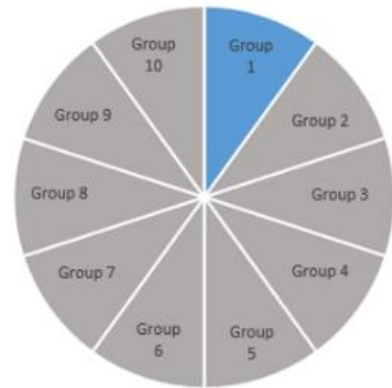

Trial 1

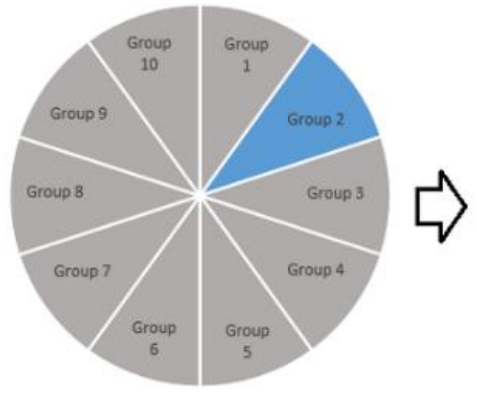

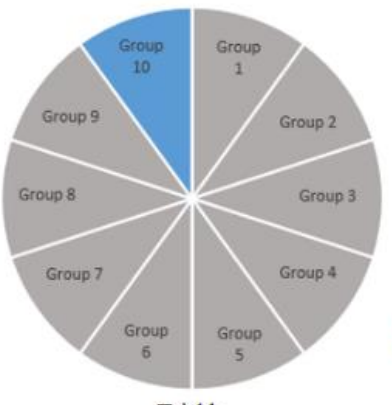

Trial 2

Trial k

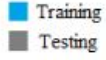

Figure 4. K-Fold Cross Validation Scheme

The implementation process is started by obtaining the histogram of the image dataset. The histogram of an image is obtained by quantizing image colors and counting the number of pixels in each color [15]. Since the image from dataset are greyscale images then the histogram result is provided the frequency of 256 grey level of each picture. This gray level is used as a feature for training process of SVM. Next step is training process of SVM. The application trained ten classes as the number of dataset class. At this process, SVM algorithm modified with multi kernel which is the kernel is obtained by combining Polynomial kernel, RBF kernel and Linear kernel. Since SVM can only classify two classes then in order to be able to classify more classes, it is modified with one-against-all method. The process of the method is suppose give $N$ classes case, then train N SVM classifier, which is the $N$-th classifier is trained by all sample dataset of class $N$ that considered as positive class while the other sample are considered as negative [12]. The testing process is started by testing the images data with SVM classifier that has been set up earlier. Then, the application will assign either the image data test is counted as "number one", "number two" class or other classes.

\subsection{Experimental Result}

The performance of the application will be delivered by computing value of Accuracy, Precision and Recall. Accuracy is defined by the degree of proximity between the predicted value and the true value, whereas Precision is defined as a calculation of the level of accuracy between the information requested by the user and the answer given by the system, meanwhile Recall is the success rate of the system in rediscovering information [16]. As a consequence, to get better understanding, this paper is also provide the result of performance application that has been built with SVM algorithm but using single kernel function, that is Polynomial order 3 and RBF single kernel. The average result of all application performance trials are presented by the following Table 1, 2, 3.

The result shows that SVM with multi kernel can recognize handwritten numerical Arabic character well. It is clear that the average of Accuracy of this application for recognizing all kind of character is about $86.63 \%$ while the average of Precision is $40.02 \%$ and $32.87 \%$ for average of Recall. The best result belongs to Number One character, which is mean that character handwritten is recognized very well by the application. It is caused the shape of Number One character is the simplest and most different among others which is only a straight vertical line.

Table result also shows the comparison between the application using multi kernel and the application using single kernel, particularly using only Polynomial kernel or RBF kernel. Overall, the result shows that the application using multi kernel method provide better result than method with single kernel RBF or Polynomial. In addition, the application using RBF kernel has the lowest performance, although as the result showed the average Accuracy value of it is $86.84 \%$ which is measly higher than others, the number of average value of its Precision and Recall shows in contrast, which is the average of its Precision is only $0.62 \%$ and average of Recall is $4.51 \%$. It is clearly show that the consistency of result provided by RBF kernel based application is extremely bad. Thus, RBF kernel is not suitable to be used in term of recognize the handwritten Arabic 
numeral character specifically using the character that obtained from MADBase which is used in this framework. In other hand, the application that has been built with single Polynomial kernel present the average value of Precision and Recall of it is $35.92 \%$ and $29.44 \%$ which mean it is slightly better than RBF kernel but still lower than the result of proposed method.

Table 1. Average Accuracy of the Application (\%)

\begin{tabular}{cccc}
\hline Class/Number & $\begin{array}{c}\text { Multi Kernel } \\
\text { Learning }\end{array}$ & $\begin{array}{c}\text { Polynomial Kernel } \\
\text { Learning }\end{array}$ & $\begin{array}{c}\text { RBF Kernel } \\
\text { Learning }\end{array}$ \\
\hline Zero & 95.32 & 94.56 & 90.00 \\
One & 98.48 & 98.38 & 90.00 \\
Two & 89.38 & 87.80 & 90.00 \\
Three & 89.98 & 89.56 & 90.00 \\
Four & 90.00 & 89.94 & 90.00 \\
Five & 92.58 & 90.26 & 90.00 \\
Six & 89.62 & 89.52 & 90.00 \\
Seven & 89.46 & 89.28 & 90.00 \\
Eight & 44.84 & 44.40 & 61.60 \\
Nine & 85.86 & 85.58 & 40.56 \\
\hline Average off All & 86.63 & 85.97 & 86.84 \\
\hline
\end{tabular}

Table 2. Average Precision of the Application (\%)

\begin{tabular}{cccc}
\hline Class/Number & $\begin{array}{c}\text { Multi Kernel } \\
\text { Learning }\end{array}$ & $\begin{array}{c}\text { Polynomial Kernel } \\
\text { Learning }\end{array}$ & $\begin{array}{c}\text { RBF Kernel } \\
\text { Learning }\end{array}$ \\
\hline Zero & 88.15 & 80.52 & 0.00 \\
One & 94.45 & 93.99 & 0.00 \\
Two & 0.00 & 2.93 & 0.00 \\
Three & 0.00 & 0.00 & 0.00 \\
Four & 6.67 & 6.67 & 0.00 \\
Five & 92.56 & 71.67 & 0.00 \\
Six & 48.88 & 46.11 & 0.00 \\
Seven & 15.05 & 6.99 & 0.00 \\
Eight & 14.46 & 14.36 & 5.56 \\
Nine & 30.08 & 29.23 & 12.43 \\
\hline Average off All & 40.02 & 35.92 & 0.62 \\
\hline
\end{tabular}

Table 3. Average Recall of the Application (\%)

\begin{tabular}{cccc}
\hline Class/Number & $\begin{array}{c}\text { Multi Kernel } \\
\text { Learning }\end{array}$ & $\begin{array}{c}\text { Polynomial Kernel } \\
\text { Learning }\end{array}$ & $\begin{array}{c}\text { RBF Kernel } \\
\text { Learning }\end{array}$ \\
\hline Zero & 62.20 & 60.80 & 0.00 \\
One & 90.40 & 89.80 & 0.00 \\
Two & 0.00 & 0.80 & 0.00 \\
Three & 0.00 & 0.00 & 0.00 \\
Four & 0.40 & 0.40 & 0.00 \\
Five & 28.20 & 3.00 & 0.00 \\
Six & 22.00 & 17.80 & 0.00 \\
Seven & 1.40 & 1.20 & 0.00 \\
Eight & 91.20 & 91.20 & 40.60 \\
Nine & 31.80 & 31.40 & 70.20 \\
\hline Average off All & 32.87 & 29.44 & 4.51 \\
\hline
\end{tabular}

\section{Conclusion}

In this framework, the handwritten Arabic numeral character recognition application using SVM algorithm modified with multi kernel method is built. Dataset that has been used in this framework taken from MADBase dataset from The American University in Cairo. This study used 5000 images containing handwritten numerical zero to nine in Arabic character. Regarding the validation, $k$-fold cross validation method is used by dividing all the dataset into ten groups and 
all of these groups is using for train and test alternately. The output shows that the proposed method provide good performance proved by the value of its Accuracy, Precision and Recall.

This framework also compares the application that is proposed with application that using only single kernel RBF or Polynomial. Moreover, the result of comparison present that handwritten Arabic numeral character recognition using multi kernel SVM is better than using single kernel SVM with the same process and dataset. The outcome also shows that using RBF kernel is not suitable to recognize handwritten Arabic numeral character especially with the dataset used in this framework. As a result, it is clear that not every kernel deliver the best output for all case, that is why determine kernel for every classification problem is not easy. Therefore, the more flexible kernel such as Multi Kernel Method is preferably chosen.

\section{References}

[1] Q, ye and D. Doerman, "Tect Detection and Recognition in Imagery: A Survey,". IEEE Transactions on Pattern Analysisi and Machine Intelligence., Vol. 37, No. 7, Pp. 1480-1500, 2015.

[2] Sivani and BS. Dipti, "Techniques of Text Detection and Recognition: A Survey," International Journal of Emerging Research in Management \& Technology., Vol. 6, No. 6, Pp. 83-87, 2017.

[3] M. Shatnawi, "Off-line Handwritten Arabic Character Recognition : A Survey," in Int'l Conf IP, Comp Vision, Pattern Recognit | IPCV'15 | - Proceeding, Pp. 52-58, 2015.

[4] T. L. Dimond, "Devices for Reading Handwritten Characters," in IRE-ACM-AIEE '57 (Eastern) Papers and discussions - Proceeding, Pp. 232-237, 1957.

[5] M.A. Mudhsh and R. Amodfer, "Arabic Handwritten Alphanumeric Character Recognition Using Very Deep Neural Network," MDPI Information Journal., Vol. 8, No. 3, Pp. 1-14, 2017.

[6] K. Jayech, M.A Mahjoub, and N.E.B. Amara, "Synchronous Multi-Stream Hidden Markov Model for Offline Arabic Handwriting Recognition Without Explicit Segmentation," Elsevier Journal of Neurocomputing., Vol. 214, Pp. 958-971, 2016.

[7] M. Shatnawi and S. Abdallah, "Improving Handwritten Arabic Character Recognition by Modeling Human Handwriting Distortions". ACM Transactions on Asian and Low-Resources Language Information Processing., Vol. 15, No. 1, Pp. 1-12, 2016

[8] R. Mouhcine, A. Mustapha, and M. Zouhir, "Recognition of Cursive Arabic Handwritten Text Using Embedded Training Based on HMMs". Elsevier Journal of Electrical Systems and Information Technology., Vol. 5, No. 2, Pp. 245-251, 2018.

[9] M. Athoillah, M.I. Irawan, and E.M. Imah, "Study Comparison of SVM-, K-NN- and Backpropagation-Based Classifier for Image Retrieval," Jurnal IImu Komputer dan Informasi (Journal of Computer Science and Information)., Vol. 8, No. 1, Pp. 11-19, 2015.

[10] B. Scholkopf and A. J. Smola, Learning with Kernels. MIT Press, 2001.

[11] A. Rakotomamonjy, R.B. Francis, S. Canu, and G. Yves, "SimpleMKL," Journal of Machine Learning Research., Vol. 9, Pp. 2491-2521, 2008.

[12] M. Athoillah, M.I. Irawan, and E.M. Imah, "Support Vector Machine with Multiple Kernel Learning for Image Retrieval," in 2015 International Conference on Information \& Communication Technology and Systems (ICTS) - Proceeding, Pp. 17-22, 2015.

[13] C. Campbell and Y. Ying, Learning with Support Vector Machines. Morgan \& Claypool Publishers, 2011.

[14] S. Abdleazeem, and E. El-Sherif, "Arabic Handwritten Higit Recognition" International Journal of Document Analysis and Recognition (IJDAR)., Vol. 11, No. 3, Pp. 127-141.

[15] A.R. Kumar, and D. Saravanan, "Content Based Image Retrieval Using Color Histogram," International Journal of Computer Science and Information Technologies., Vol. 4, No. 2, Pp. 242-245, 2013.

[16] S. Abe. Introduction in Support Vector Machines for Pattern Classification. Springer, London, 2010. 
